\title{
Comparación de los resultados de manejo laparoscópico del embarazo ectópico con el uso de energía bipolar avanzada versus híbrida (ultrasónica más bipolar)
}

\author{
Comparison of the results of laparoscopic management of ectopic pregnancy \\ with the use of advanced bipolar energy versus hybrid (ultrasonic plus bipolar) \\ Blanca Fátima Ayala Pacheco, ${ }^{*}$ Consuelo Hernández Álvarez ${ }^{\ddagger}$ \\ Citar como: Ayala PBF, Hernández ÁC. Comparación de los resultados de manejo laparoscópico del embarazo ectópico con el uso de \\ energía bipolar avanzada versus híbrida (ultrasónica más bipolar). Acta Med. 2021; 19 (1): 9-14. https://dx.doi.org/10.35366/98563
}

\section{Resumen}

Objetivo: Comparar los resultados del manejo laparoscópico de embarazo ectópico con el uso de energía bipolar avanzada versus híbrida. Material y métodos: Se realizó un estudio observacional, descriptivo, tipo serie de casos, a partir de expedientes de pacientes con embarazo ectópico tubárico confirmado. Estas pacientes fueron sometidas a salpingectomía laparoscópica usando energía bipolar avanzada o híbrida (ultrasónica más bipolar) en el Hospital Ángeles Pedregal durante el periodo de 2013 a 2019. Se compararon desenlaces entre grupos y se consideró significativa una $p<0.05$. Resultados: Se atendieron 101 casos de embarazo ectópico. En 60 de 101 procedimientos fue usada energía híbrida (59.4\%) y en los 41 restantes bipolar avanzada $(40.6 \%)$. En pacientes con embarazo ectópico roto la tasa de complicaciones en el grupo energía híbrida fue de 8.4 versus $7.1 \%$ en el grupo energía bipolar $(p=0.760)$. En pacientes con embarazo ectópico no roto la tasa de complicaciones fue de $3.7 \%$ en el grupo energía bipolar $(p=0.429)$. Las pacientes del grupo energía híbrida tuvieron una reducción significativa en las cifras de hemoglobina en el postoperatorio $(p=0.011)$. Conclusión: El uso de energía híbrida se asocia con mayor reducción de hemoglobina durante el postoperatorio sin incrementar otras complicaciones ni estancia hospitalaria en pacientes con embarazo ectópico.

Palabras clave: Salpingectomía, embarazo ectópico, energía híbrida y bipolar.

\section{Abstract}

Objective: To compare the results of laparoscopic management of ectopic pregnancy with the use of advanced bipolar versus hybrid energy. Material and methods: An observational, descriptive, case series study was conducted, based on records of patients with tubal ectopic pregnancy confirmed. These patients underwent laparoscopic salpingectomy using advanced bipolar or hybrid energy (ultrasonic plus bipolar) at the Hospital Angeles Pedregal during 2013 to 2019. Outcomes were compared between groups considering significant a $p$ value $<0.05$. Results: In total 101 cases of ectopic pregnancy were treated. In 60 out of 101 procedures, hybrid energy $(59.4 \%)$ was used and in 41 advanced bipolar energy was employed (40.6\%). In patients with ruptured ectopic pregnancy the complication rate in the hybrid energy group was 8.4 versus $7.1 \%$ in the bipolar energy group $(p=0.760)$. In patients with not-ruptured ectopic pregnancy the complication rate was $0 \%$ in the hybrid energy group and $3.7 \%$ in the bipolar energy group $(p=0.429)$. Patients in the hybrid energy group had a significant reduction in hemoglobin levels after the operation ( $p$ $=0.011$ ). Conclusion: The use of hybrid energy is associated with greater hemoglobin reduction during the postoperative period without increasing other complications or hospital stay in patients with ectopic pregnancy.

Keywords: Salpingectomy, ectopic pregnancy, hybrid and bipolar energy.

\footnotetext{
* Médico Residente de Ginecología y Obstetricia. Facultad Mexicana de Medicina de la Universidad La Salle.

‡ Departamento de Ginecología y Obstetricia. Hospital Ángeles Acoxpa.

Correspondencia:

Dra. Blanca Fátima Ayala Pacheco

Correo electrónico: dra.fatima.ayala@gmail.com

Aceptado: 12-12-2019.

Hospital Ángeles Pedregal. Ciudad de México, México.
}

www.medigraphic.com/actamedica 


\section{INTRODUCCIÓN}

El embarazo ectópico se define como la implantación del huevo fecundado fuera de la cavidad uterina. ${ }^{1}$ A nivel global tiene una incidencia de $2 \%$ y ésta aumenta con la edad de la mujer; ${ }^{2}$ en México la incidencia varía, pero se estima que es de $1.6-2.0 \%{ }^{3}$

Entre los antecedentes quirúrgicos de riesgo se han reportado los siguientes: cirugía tubárica previa, patología tubárica, embarazo ectópico previo, cesárea previa, aborto previo, infertilidad y cirugía abdominal/pélvica previa. ${ }^{4,5}$

Los principales sitios de ocurrencia del ectópico son las trompas de Falopio en 98\% de los casos, y el restante ocurre en el sitio de cicatrices uterinas, abdominal, ovario y rara vez en cérvix. De los que ocurren en la trompa, $70 \%$ se localizan en la ámpula, $11 \%$ en el istmo, $12 \%$ en la fimbria y $3 \%$ en la porción intersticial. ${ }^{6}$ El diagnóstico de embarazo ectópico se establece mediante ultrasonografía transvaginal y la determinación en sangre de hCG- $\beta$. ${ }^{7}$

El manejo del embarazo ectópico es médico y quirúrgico, el abordaje laparoscópico es el estándar de oro por sus ventajas en términos de invasividad, tiempo de recuperación y menor morbilidad. ${ }^{8-10}$ Sin embargo, no está claro qué modalidad de energía para corte y coagulación es la más apropiada, si la ultrasónica, la bipolar avanzada o la híbrida; estas técnicas revolucionarias han reemplazado a la energía monopolar clásica y a la bipolar convencional. ${ }^{11}$

La energía bipolar avanzada, como la proporcionada a través de las pinzas ENSEAL, permite la disecación, coaptación y transección tisular, pero tiene una capacidad limitada para cortar y coagular grandes áreas de sangrado porque la corriente electroquirúrgica está restringida al área de contacto del electrodo. Mientras que la ultrasónica ofrece también disecación y coaptación, pero con una transección tisular mecánica rápida, y la energía híbrida (Thunderbeat) al combinar la ultrasónica más bipolar ofrece el corte rápido de tejidos mediante la energía ultrasónica y la capacidad para sellar vasos sanguíneos de forma segura mediante energía bipolar. ${ }^{12-14}$

Algunos estudios previos han comparado distintas modalidades de energía para disección, coaptación, vaporización y transección, incluyendo ENSEAL versus estándar bipolar, ENSEAL versus LigaSure o versus Plasma Kinetic Gyrus durante distintos procedimientos laparoscópicos, pero hasta nuestro conocimiento no se han realizado comparaciones de ENSEAL versus Thunderbeat. ${ }^{15}$

En el presente estudio se comparan los resultados de manejo laparoscópico de embarazo ectópico con el uso de energía bipolar avanzada versus híbrida (ultrasónica más bipolar), mediante el análisis de una serie de casos del Hospital Ángeles Pedregal.

Tabla 1: Comparación de características clínicas y tasa de embarazo roto entre grupos.

Característica

Edad materna (años)

Edad gestacional (semanas)

Niveles de hCG- $\beta$

Eventos obstétricos

Gestas

Paras

Cesáreas

Antecedentes quirúrgicos, \% (n)

Cesárea previa

Legrado instrumentado

Apendicectomía

Amigdalectomía

Salpingostomía

Abortos provocados

Colecistectomía

Otros obstétricos

Antecedente de ectópico, \% (n)

Molas previas, \% (n)

Hemoglobina pre-qx $(\mathrm{g} / \mathrm{dL})$

Hematocrito pre-qx ( $\mathrm{g} / \mathrm{dL})$

Embarazo ectópico roto, \% (n)

Energía híbrida $(n=60)$

$\begin{aligned} 32.4 & \pm 4.9 \\ 6.8 & \pm 1.9 \\ 210.8 & \pm 5,198.8\end{aligned}$

$2.0 \pm 1.2$

$1.9 \pm 0.2$

$1.8 \pm 0.4$

$71.7(43)$

31.7 (19)

$16.7(10)$

$8.3(5)$

$3.3(2)$

$10.0(6)$

$5.0(3)$

$6.7(4)$

$5.0(3)$

$10.0(6)$

1.7 (1)

$12.9 \pm 11.7$

$37.7 \pm 4.6$

$76.9(10)$
Energía bipolar avanzada $(n=41)$

p

$\begin{array}{cc}32.0 \pm 5.1 & 0.670 \\ 6.8 \pm 1.9 & 0.933 \\ 4,309.0 \pm 3,989.1 & 0.959 \\ & \\ 1.8 \pm 1.1 & 0.245 \\ 1.8 \pm 0.4 & 0.129 \\ 1.8 \pm 0.4 & 0.963 \\ 58.5(24) & 0.201 \\ 22.0(9) & 0.284 \\ 14.6(6) & 0.784 \\ 2.4(1) & 0.397 \\ 9.8(4) & 0.220 \\ 4.9(2) & 0.468 \\ 2.4(1) & 0.645 \\ 2.4(1) & 0.408 \\ 2.4(1) & 0.767 \\ 4.9(2) & 0.468 \\ 2.4(1) & 1.000 \\ 13.3 \pm 1.2 & 0.094 \\ 39.3 \pm 4.5 & 0.095 \\ 16.7(1) & 0.041\end{array}$


Tabla 2: Comparación de características quirúrgicas entre grupos, en aquellos con y sin embarazo ectópico roto.

\begin{tabular}{|c|c|c|c|c|c|c|}
\hline \multirow[b]{2}{*}{ Característica } & \multicolumn{3}{|c|}{ Ectópico roto } & \multicolumn{3}{|c|}{ Ectópico no roto } \\
\hline & $\begin{array}{l}\text { Energía híbrida } \\
\qquad(n=24)\end{array}$ & $\begin{array}{l}\text { Energía bipolar } \\
\text { avanzada }(n=14)\end{array}$ & $p$ & $\begin{array}{l}\text { Energía híbrida } \\
\qquad(n=36)\end{array}$ & $\begin{array}{c}\text { Energía bipolar } \\
\text { avanzada }(n=27)\end{array}$ & $p$ \\
\hline \multicolumn{7}{|l|}{ Localización de ectópico, \% (n) } \\
\hline Ampular derecho & $58.3(14)$ & $35.7(5)$ & & $44.4(16)$ & $51.9(14)$ & \\
\hline Ampular izquierdo & $20.8(5)$ & $35.7(5)$ & 0.450 & $36.1(13)$ & $25.9(7)$ & \\
\hline Infundibular derecho & $16.7(4)$ & $28.6(4)$ & & $2.8(1)$ & $3.7(1)$ & 0.603 \\
\hline Infundibular izquierdo & $4.2(1)$ & $0.0(0)$ & & $13.9(5)$ & $11.1(3)$ & \\
\hline Ístmico derecho & $0.0(0)$ & $0.0(0)$ & & $0.0(0)$ & $7.4(2)$ & \\
\hline Ístmico izquierdo & $0.0(0)$ & $0.0(0)$ & & $2.8(1)$ & $0.0(0)$ & \\
\hline \multicolumn{7}{|l|}{ Transfusiones, \% (n) } \\
\hline Plasma fresco congelado & $0.0(0)$ & $0.0(0)$ & 1.000 & $2.8(1)$ & $0.0(0)$ & 1.000 \\
\hline Paquete globular & $8.3(2)$ & $14.3(2)$ & 0.616 & $13.9(5)$ & $7.4(2)$ & 0.689 \\
\hline Hemoperitoneo & $95.8(23)$ & $100.0(14)$ & 1.000 & $0.0(0)$ & $0.0(0)$ & 1.000 \\
\hline Tiempo quirúrgico (minutos) & $108.5 \pm 49.3$ & $95.1 \pm 32.5$ & 0.370 & $83.7 \pm 31.4$ & $85.5 \pm 32.3$ & 0.831 \\
\hline Volumen de hemoperitoneo $(\mathrm{mL})$ & $413.9 \pm 374.8$ & $262.9 \pm 261.1$ & 0.195 & $0.0 \pm 0.0$ & $0.0 \pm 0.0$ & 1.000 \\
\hline $\begin{array}{l}\text { Sangrado transquirúrgico sin } \\
\text { hemoperitoneo }\end{array}$ & $168.7 \pm 129.2$ & $132.1 \pm 72.3$ & 0.338 & $190.4 \pm 148.7$ & $147.9 \pm 28.5$ & 0.447 \\
\hline
\end{tabular}

\section{MATERIAL Y MÉTODOS}

Se realizó un estudio observacional, descriptivo, tipo serie de casos, a partir de expedientes de pacientes que fueron atendidas por embarazo ectópico (corroborado sérica y ultrasonográficamente) en el Hospital Ángeles Pedregal durante el periodo de enero de 2013 a enero de 2019. Estas pacientes fueron manejadas por vía laparoscópica usando energía híbrida (con pinzas Thunderbeat, Olympus Medical System) o energía bipolar avanzada (con pinzas ENSEAL Ethicon, Johnson). No se excluyeron pacientes para evitar sesgo de selección de casos.

En todos los casos se realizó el abordaje laparoscópico convencional para hueco pélvico: paciente en posición de Trendelenburg, con colocación de movilizador uterino (pinza de Hulka o cánula de Kroener) con miembros pélvicos semiflexionados; abordaje laparoscópico cerrado con aguja de Veress y colocación de trocar transumbilical, en ambas fosas iliacas y otro suprapúbico. Posterior a creación de neumoperitoneo y visualización de cavidad pélvica, se aspira y se cuantifica sangre y coágulos (en caso de hemoperitoneo), con identificación subsecuente del saco gestacional en tuba uterina, pinzamiento de porción distal de la salpinge con pinza atraumática de forma que se exponga el mesosálpinx, coagulación con pinza bipolar o híbrida y posterior corte de la salpinge a $1 \mathrm{~cm}$ de la porción cornual hasta su liberación y extracción transumbilical. Finalmente, previo a la liberación del neumoperitoneo y extracción de trócares, se realiza lavado de cavidad y hemostasia. En los casos requeridos se realiza adherenciólisis previa a salpingectomía con el mismo tipo de energía.

Se obtuvo información clínica y quirúrgica relevante de los expedientes electrónicos, incluyendo los desenlaces principales de interés: hemoglobina, hematocrito, sangrado, estancia hospitalaria, puntaje de dolor postoperatorio y complicaciones transoperatorias.

Este estudio toma en cuenta las consideraciones éticas para investigación en seres humanos de la declaración de Helsinki, del código de Núremberg y de la Ley General en Salud de los Estados Unidos Mexicanos.

El análisis estadístico se realizó en SPSS versión 25 para Mac, en el cual se realizaron pruebas estadísticas descriptivas consistentes en media y desviación estándar para variables cuantitativas, frecuencias y porcentajes para variables cualitativas. Como pruebas estadísticas inferenciales se utilizaron $\chi^{2}$ y exacta de Fisher para variables cualitativas y t de Student para variables cuantitativas. Una $p<0.05$ se consideró significativa.

\section{RESULTADOS}

Se atendieron un total de 101 pacientes por embarazo ectópico durante el periodo de estudio. Sesenta de 101 procedimientos se realizaron con energía híbrida (59.40\%) y 41 con energía bipolar (40.59\%). La mayoría de las características clínicas fueron significativas entre grupos, 
solamente las pacientes que fueron operadas con energía híbrida tuvieron mayor frecuencia de embarazo ectópico roto $(76.9 \%$ versus $16.7 \%, p=0.041$ ) (Tabla 1 ).

Por lo anterior, las comparaciones posteriores se realizaron por separado en pacientes con embarazo ectópico roto y no roto. No hubo diferencias significativas en las características quirúrgicas entre grupos en pacientes con embarazo ectópico roto ni en pacientes con embarazo ectópico no roto (Tabla 2). Sin embargo, al comparar los resultados y desenlaces quirúrgicos entre grupos, se encontraron algunas diferencias significativas.

En pacientes con embarazo ectópico roto la tasa de complicaciones en el grupo energía híbrida fue de $8.4 \%$ versus $7.1 \%$ en el grupo energía bipolar $(p=0.760)$. La hemoglobina postoperatoria fue significativamente menor en el grupo energía híbrida (10.6 $\pm 1.5 \%$ ) que en el grupo energía bipolar $(11.8 \pm 1.7 \%, \mathrm{p}=0.030)$ y el hematocrito postoperatorio también tendió a ser menor. En otros parámetros no hubo diferencias significativas entre grupos.

En las pacientes con embarazo ectópico no roto la tasa de complicaciones fue de $0 \%$ en el grupo energía híbrida y de $3.7 \%$ en el grupo energía bipolar $(p=0.429)$. Las pacientes del grupo energía híbrida tuvieron una reducción significativamente mayor en las cifras de hemoglobina en el postoperatorio $(p=0.011)$ y por lo tanto menores valores de hemoglobina en el postoperatorio que las pacientes del grupo energía bipolar ( $p=0.047$ ) (Tabla 3 ).

\section{DISCUSIÓN}

En la presente serie de casos se resume la experiencia con el uso de energía híbrida versus bipolar durante el manejo laparoscópico de embarazo ectópico en el Hospital Ángeles Pedregal. En primer lugar, se encontró que tres cuartas partes de las pacientes tratadas con energía híbrida tuvieron embarazo ectópico roto en comparación con menos de una quinta parte en las pacientes del grupo energía bipolar. Por lo anterior, y dado que el ectópico roto se asocia a menudo con dolor abdominal intenso, sangrado vaginal y hemorragia interna, ${ }^{16}$ el resto de las comparaciones se realizaron por separado en quienes presentaron embarazo ectópico roto y no roto.

Encontramos que las pacientes operadas con energía híbrida y bipolar avanzada con y sin ectópico roto fueron muy similares en edad gestacional, localización del embarazo ectópico, cifras de hemoglobina y hematocrito prequirúrgicas. Sin embargo, en el postoperatorio las pacientes del grupo energía híbrida tuvieron significativamente menores cifras de hemoglobina independientemente de presentar o no embarazo ectópico roto. Asimismo, en el grupo embara-

Tabla 3: Comparación de resultados/desenlaces quirúrgicos entre grupos.

Ectópico roto

\begin{tabular}{|c|c|c|c|c|c|c|}
\hline \multirow[b]{2}{*}{ Característica } & & \\
\hline & $\begin{array}{l}\text { Energía híbrida } \\
\qquad(n=24)\end{array}$ & $\begin{array}{c}\text { Energía bipolar } \\
\text { avanzada }(n=14)\end{array}$ & $p$ & $\begin{array}{l}\text { Energía híbrida } \\
\qquad(n=36)\end{array}$ & $\begin{array}{c}\text { Energía bipolar } \\
\text { avanzada }(n=27)\end{array}$ & $p$ \\
\hline Complicaciones, \% (n) & & & & 0.0 & $3.7(1)$ & \\
\hline Choque anafiláctico & $0.0(0)$ & $0.0(0)$ & & $0.0(0)$ & $0.0(0)$ & \\
\hline Choque hipovolémico & $0.0(0)$ & $7.1(1)$ & & $0.0(0)$ & $0.0(0)$ & \\
\hline Perforación intestinal resuelta & $4.2(1)$ & $0.0(0)$ & 0.760 & $0.0(0)$ & $0.0(0)$ & 0.429 \\
\hline Perforación uterina & $4.2(1)$ & $0.0(0)$ & & $0.0(0)$ & $0.0(0)$ & \\
\hline Ninguna & $91.7(22)$ & $92.9(13)$ & & $100.0(0)$ & $96.3(0)$ & \\
\hline Total sangrado & $581.3 \pm 397.5$ & $395.0 \pm 288.3$ & 0.137 & $190.4 \pm 148.7$ & $165.2 \pm 146.3$ & 0.505 \\
\hline Hemoglobina pre-qx (g/dL) & $12.1 \pm 1.9$ & $13.0 \pm 1.5$ & 0.122 & $13.4 \pm 1.3$ & $13.5 \pm 1.1$ & 0.637 \\
\hline Hemoglobina post-qx (g/dL) & $10.6 \pm 1.5$ & $11.8 \pm 1.7$ & 0.030 & $11.9 \pm 1.3$ & $12.6 \pm 1.3$ & 0.047 \\
\hline Hematocrito pre-qx (g/dL) & $35.6 \pm 4.7$ & $38.3 \pm 4.3$ & 0.089 & $39.1 \pm 4.1$ & $39.8 \pm 4.6$ & 0.549 \\
\hline Hematocrito post-qx (g/dL) & $33.8 \pm 3.8$ & $36.2 \pm 3.9$ & 0.073 & $36.5 \pm 3.9$ & $37.7 \pm 3.9$ & 0.227 \\
\hline Cambio en hemoglobina ( $\mathrm{g} / \mathrm{dL})$ & $-1.5 \pm 0.9$ & $-1.2 \pm 0.8$ & 0.373 & $-1.4 \pm 0.9$ & $-0.9 \pm 0.6$ & 0.011 \\
\hline Cambio en hematocrito (\%) & $-1.7 \pm 2.6$ & $-2.0 \pm 1.4$ & 0.700 & $-2.6 \pm 1.9$ & $-2.0 \pm 1.4$ & 0.213 \\
\hline Estancia hospitalaria (días) & $2.2 \pm 1.2$ & $1.9 \pm 0.8$ & 0.507 & $1.7 \pm 0.6$ & $1.6 \pm 0.6$ & 0.495 \\
\hline \multicolumn{7}{|l|}{ Puntaje EVA de dolor } \\
\hline 2 horas & $3.3 \pm 1.9$ & $4.1 \pm 2.4$ & 0.242 & $3.9 \pm 2.2$ & $4.0 \pm 2.6$ & 0.771 \\
\hline 24 horas & $2.0 \pm 1.8$ & $2.0 \pm 1.9$ & 0.947 & $2.3 \pm 1.9$ & $1.8 \pm 1.4$ & 0.227 \\
\hline 48 horas & $2.3 \pm 1.8$ & $2.5 \pm 2.1$ & 0.799 & $2.7 \pm 2.1$ & $3.0 \pm 2.3$ & 0.671 \\
\hline
\end{tabular}


zo ectópico no roto el cambio en las cifras de hemoglobina fue significativamente mayor en los operados con energía híbrida que con energía bipolar avanzada. Por lo tanto, en este desenlace las pacientes operadas con energía bipolar avanzada tuvieron mejores resultados.

No obstante, en relación con las complicaciones transquirúrgicas, no hubo diferencias significativas entre grupos en choque anafiláctico, choque hipovolémico, perforación intestinal y perforación uterina entre los operados con energía híbrida y bipolar avanzada. Tampoco se encontraron diferencias significativas entre grupos en el puntaje de dolor en las 48 horas postoperatorias ni en la estancia hospitalaria o en el sangrado total estimado.

No existen estudios previos en cirugía laparoscópica ginecológica en que se haya comparado la experiencia de manejo quirúrgico con energía híbrida versus bipolar avanzada; sin embargo, un estudio realizado en un modelo porcino encontró que el uso de energía híbrida Thunderbeat presentaba mayor versatilidad que otros dispositivos incluyendo ENSEAL y LigaSure dado que proporcionaba una disección más rápida, presión de estallido similar, capacidad para realizar hemostasia y una aceptable diseminación térmica, presentando a esta tecnología como segura para cortar, coagular y disecar tejido, que reduce el tiempo quirúrgico. ${ }^{17}$ Sin embargo, en nuestro estudio no se asoció con menor tiempo quirúrgico, pero sí con una mayor disminución de hemoglobina postoperatorias en pacientes con ectópico no roto. Incluso, aunque no hubo diferencia estadística significativa, las pacientes con embarazo ectópico roto mostraron una tendencia a tener mayor volumen de sangrado $581 \mathrm{~mL}$ versus $391 \mathrm{~mL}$ en promedio.

En una comparación de energía híbrida (Thunderbeat) con electrocirugía estándar en histerectomía y linfadenectomía laparoscópica, aunque se demostró una reducción del tiempo quirúrgico y en el puntaje de dolor con el uso de la primera, no se encontraron diferencias en la pérdida sanguínea ni en complicaciones o estancia hospitalaria. ${ }^{18} \mathrm{No}$ obstante, no encontramos en nuestro estudio diferencias significativas en dolor ni en tiempo quirúrgico al comparar la energía híbrida con bipolar avanzada (ENSEAL).

En lo que respecta a energía bipolar avanzada (ENSEAL), aunque se han reportado ventajas en comparación con la energía bipolar estándar en términos de sangrado y estancia hospitalaria, ha demostrado ser menos buena en términos de pérdida sanguínea que la energía cinética con plasma (PK) en pacientes sometidas a histerectomía subtotal o total laparoscópica. ${ }^{19,20}$ Sin embargo, no se encontraron comparaciones de energía bipolar avanzada (ENSEAL) con energía híbrida (Thunderbeat).

Hasta nuestro conocimiento, el presente estudio parece ser el primero en presentar y comparar la experiencia de manejo quirúrgico laparoscópico de embarazo ectópico roto y no roto con energía híbrida (Thunderbeat) y bipolar avanzada (ENSEAL).

Si bien se trata de un estudio observacional, retrospectivo que no permite establecer una relación causa-efecto y que tiene limitantes debido a la obtención de información de forma retrospectiva, los resultados presentados son valiosos dado que la casuística acumulada es buena y aporta una pieza más al conocimiento sobre el uso de pinzas de nueva generación para coagulación, disección y corte en cirugía endoscópica ginecológica.

Por el momento, con base en el presente estudio, es preferible el uso de energía bipolar avanzada (ENSEAL) hasta que no haya información nueva y robusta.

\section{CONCLUSIÓN}

El uso de energía híbrida se asoció con mayor reducción de hemoglobina durante el transoperatorio que el uso de energía bipolar avanzada sin incrementar otras complicaciones ni la estancia hospitalaria, por lo que se recomienda el uso de energía bipolar avanzada hasta que no exista nueva evidencia disponible y el monitoreo cuidadoso de la hemoglobina postoperatoria en las pacientes operadas con energía híbrida.

\section{REFERENCIAS}

1. Park JE, Yuk JS, Cho IA, Baek JC, Lee JH, Park JK. Ectopic pregnancy incidence in the Republic of Korea in 2009-2015: A population-based cross-sectional study. Sci Rep. 2018; 8 (1): 17308.

2. Newbatt E, Beckles Z, Ullman R, Lumsden MA; Guideline Development Group. Ectopic pregnancy and miscarriage: summary of NICE guidance. BMJ. 2012; 345: e8136. doi: 10.1136/bmj.e8136.

3. Escobar-Padilla B, Perez-López CA, Martínez-Puon H. Factores de riesgo y características clínicas del embarazo ectópico. Rev Med Inst Mex Seg Soc. 2017; 55 (3): 278-285.

4. Parashi S, Moukhah S, Ashrafi M. Main risk factors for ectopic pregnancy: a case-control study in a sample of Iranian women. Int J Fertil Steril. 2014; 8 (2): 147-154.

5. Gaskins AJ, Missmer SA, Rich-Edwards JW, Williams PL, Souter I, Chavarro JE. Demographic, lifestyle, and reproductive risk factors for ectopic pregnancy. Fertil Steril. 2018; 110 (7): 1328-1337.

6. Marion LL, Meeks GR. Ectopic pregnancy: history, incidence, epidemiology, and risk factors. Clin Obstet Gynecol. 2012; 55 (2): 376-386.

7. van Mello NM, Mol F, Ankum WM, Mol BW, van der Veen F, Hajenius PJ. Ectopic pregnancy: how the diagnostic and therapeutic management has changed. Fertil Steril. 2012; 98 (5): 1066-1073.

8. Duggal BS, Tarneja P, Sharma RK, Rath SK, Wadhwa RD. Laparoscopic management of ectopic pregnancies. Med J Armed Forces India. 2004; 60 (3): 220-223.

9. Alkatout I, Honemeyer U, Strauss A, Tinelli A, Malvasi A, Jonat W et al. Clinical diagnosis and treatment of ectopic pregnancy. Obstet Gynecol Surv. 2013; 68 (8): 571-581.

10. Ding DC, Chu TY, Kao SP, Chen PC, Wei YC. Laparoscopic management of tubal ectopic pregnancy. JSLS. 2008; 12 (3): 273-276.

11. Lyons SD, Law KS. Laparoscopic vessel sealing technologies. J Minim Invasive Gynecol. 2013; 20 (3): 301-307. 
12. Sankaranarayanan G, Resapu RR, Jones DB, Schwaitzberg S, De S. Common uses and cited complications of energy in surgery. Surg Endosc. 2013; 27 (9): 3056-3072.

13. Law KS, Lyons SD. Comparative studies of energy sources in gynecologic laparoscopy. J Minim Invasive Gynecol. 2013; 20 (3): 308-318.

14. Olympus Corporation. Thunderbeat plataforma de tratamiento de tejidos: Guía de consulta para profesionales sanitarios. Miami: 2018.

15. Jaiswal A, Huang KG. Energy devices in gynecological laparoscopy - Archaic to modern era. Gynecol Minim Invasive Ther. 2017; 6 (4): 147-151.

16. Dalsgaard Jensen T, Penninga L. Non-operative treatment of ruptured ectopic pregnancy. BMJ Case Rep. 2016; 2016: bcr2016215311.

17. Milsom J, Trencheva K, Monette S, Pavoor R, Shukla P, Ma J et al. Evaluation of the safety, efficacy, and versatility of a new surgical energy device (THUNDERBEAT) in comparison with Harmonic ACE, LigaSure $\mathrm{V}$, and EnSeal devices in a porcine model. J LaparoendosC Adv Surg Tech A. 2012; 22 (4): 378-386.

18. Fagotti A, Vizzielli G, Fanfani F, Gallotta V, Rossitto C, Costantini B et al. Randomized study comparing use of THUNDERBEAT technology vs standard electrosurgery during laparoscopic radical hysterectomy and pelvic lymphadenectomy for gynecologic cancer. J Minim Invasive Gynecol. 2014; 21 (3): 447-453.

19. Rothmund R, Kraemer B, Brucker S, Taran FA, Wallwiener M, Zubke A et al. Laparoscopic supracervical hysterectomy using EnSeal vs standard bipolar coagulation technique: randomized controlled trial. J Minim Invasive Gynecol. 2013; 20 (5): 661-666.

20. Aytan H, Nazik H, Narin R, Api M, Tok EC. Comparison of the use of LigaSure, HALO PKS cutting forceps, and ENSEAL tissue sealer in total laparoscopic hysterectomy: a randomized trial. J Minim Invasive Gynecol. 2014; 21 (4): 650-655. 